\title{
Gemeinwesenarbeit als demokratiefördernde Brückenbauerin
}

\author{
Milena Riede
}

\section{Vielfalt, Teilhabemöglichkeiten und soziales Miteinander}

Bei näherer Betrachtung der gesellschaftlichen Lage wird ersichtlich, dass gegenwärtige Wandlungsprozesse sozioökonomische und soziokulturelle Disparitäten innerhalb der Gesellschaft verstärken. In fast allen westlichen Gesellschaften gibt es seit Ende der 1980er-Jahre einen Trend zur Zunahme der sozialen Ungleichheit - Wachstums- und Globalisierungsdividende sind ungleich verteilt. Ein Aufklaffen der Ungleichheitsschere auf dem Arbeitsmarkt zwischen hoch und gering Qualifizierten, eine wachsende Vermögenskonzentration, festgesetzte Armut sowie abgebremste Aufstiegsmobilität lassen sich beobachten. Doch neben der sozioökonomischen Spaltungslinie und zunehmender Statusunsicherheit treten auch verstärkt soziokulturelle Spaltungslinien zutage. Durch die Globalisierungsprozesse, die Öffnung von Nationalgesellschaften, Mobilität, Migration und die Entstehung transnationaler Milieus wird das Modell von geschlossenen Nationalgesellschaften herausgefordert. Im Vorteil sind die Menschen, die flexibel und mobilitätsbereit sind, aber insbesondere die, die über hohes Humankapital verfügen (vgl. Mau 2017: 300 f.).

Seit dem Sommer 2015 bewegen das Thema Zuwanderung und die damit verbundenen Auswirkungen verstärkt die Gemüter in Deutschland und Europa. Viele Menschen sind auf der Suche nach Sicherheit, Frieden und einer besseren Zukunftsperspektive nach Deutschland gekommen, haben die Themen von Krieg, Vertreibung und Folgen des Klimawandels vor unsere eigene Haustüre getragen und Menschen damit unvermittelter konfrontiert. Die Ankunft vieler geflüchteter Menschen und die hohe mediale Aufmerksamkeit haben eine ungeahnte Aktivierungswelle in der Bevölkerung freigesetzt. Zahlreiche Menschen setzen sich seitdem für Geflüchtete ein und organisier(t)en zeitnah und unbürokratisch Netzwerke der Hilfe. Aber auch andere organisier(t)en sich, um ihre Ablehnung gegen diese Veränderungen und die Menschen mit Fluchterfahrung auszudrücken.

Unsere Gesellschaft ist durch die ankommenden Menschen vielfältiger und damit heterogener geworden, wobei die Kommunen und natürlich vor allem die Menschen vor Ort sehr unterschiedliche Vorerfahrungen im Umgang mit Vielfalt erworben haben. „Die durch Globalisierung und Pluralisierung entstandene Viel- 
falt und der professionelle Umgang mit dieser ist eine der zentralen Herausforderungen der Profession und Disziplin der Sozialen Arbeit" (AschenbrennerWellmann 2014: 8) und insbesondere ihres Handlungsfeldes der Gemeinwesenarbeit (GWA). Auf einer theoretischen Ebene konzeptualisieren Ansätze wie der Diversity-Ansatz (vgl. z. B. Leiprecht 2011) oder der Inklusionsansatz (vgl. z. B. Spatscheck \& Thiessen 2017) diese Vielfalt und plädieren für die Anerkennung und Gleichberechtigung von Verschiedenheiten. Hierbei benennt Kronauer (2010) drei zentrale Aspekte in Bezug auf ein inklusives Miteinander in der Gesellschaft, die für gesellschaftliche Teilhabe und Zugehörigkeit bedeutsam sind: Recht, Arbeit sowie soziale und familiäre Nahbeziehungen. Zahlreiche Menschen mit und ohne Migrationshintergrund sind von Teilhabe in einem oder mehreren gesellschaftlichen Bereich(en) kurz-, mittel- oder langfristig ausgeschlossen. Mithilfe digitaler Medien vernetzen sich die Menschen zwar immer mehr, allerdings zumeist mit Gleichgesinnten, wodurch das Einüben von Wechselseitigkeit und Empathie kaum stattfindet (vgl. Mau 2017: 300 f.). „Es gibt weniger Überschneidungen sozialer Kreise, man gesellt sich zu Seinesgleichen“ (Mau 2017: 302).

Angesichts der gesellschaftlichen Entwicklungen stellt sich die Frage, wie mit diesen Veränderungen umgegangen wird und wo für Menschen in ihrer Nachbarschaft nicht kommerzielle Begegnungsorte, Austausch- und Dialogmöglichkeiten vorhanden sind. Gerade auch im Kontext der Veränderungen durch Ankommende hat sich gezeigt, dass die schleppende, teilweise sehr kurzfristig, oft unzureichend oder manchmal erst im Nachhinein stattfindende Kommunikation zwischen Politik, Verwaltung und den Bewohner*innen vor Ort vielerorts für Unmut gesorgt hat. Doch wie können angesichts wachsender Polarisierung, geringer Überschneidungen sozialer Kreise bei gleichzeitig fehlenden Dialogforen Möglichkeiten gefunden werden, die ein demokratisches, soziales Miteinander lokal vor Ort verbessern?

\section{Das Handlungsfeld und Konzept der Gemeinwesenarbeit}

Bereits seit ca. 125 Jahren werden erfolgreich Methoden und Prinzipien der Gemeinwesenarbeit in Gebieten mit besonderem Entwicklungsbedarf angewandt. Die Soziale Arbeit verfügt damit über ein Handlungsfeld, das zielgruppenübergreifend und umfassend ansetzt, wobei die Wurzeln der Gemeinwesenarbeit $u$. a. in der sogenannten Settlement-Bewegung in England und den USA liegen. Sozial engagierte Menschen mit guter Bildung zogen in klassische Zuwanderungsgebiete, um sich dort gemeinsam mit den Bewohner*innen für strukturelle Verbesserungen der Lebensbedingungen einzusetzen. 
Mit „Gemeinwesen“ wird das Beziehungsgeflecht zwischen Menschen, Gruppen und Organisationen in einem umschriebenen Gebiet (z. B. Stadtteil, Kiez, Dorf) bezeichnet (vgl. Oelschlägel 2016: 50).

„Ziel ist die Verbesserung von materiellen (z. B. Wohnraum, Existenzsicherung), infrastrukturellen (z. B. Verkehrsanbindung, Einkaufsmöglichkeiten, Grünflächen) und immateriellen (z. B. Qualität sozialer Beziehungen, Partizipation, Kultur) Bedingungen unter maßgeblicher Einbeziehung der Betroffenen“"(Stövesand \& Stoik 2013: 21).

Angesichts dieser großen und weit gefassten Ziele ist es offensichtlich, dass diese nur in einem langfristigen Prozess und nur in einem interdisziplinären Kooperationsprozess erreicht werden können.

\subsection{Städtebauförderprogramm Soziale Stadt (Quartiersmanagement) und Gemeinwesenarbeit}

Bereits seit den 1980er-Jahren lassen sich eine verstärkte sozialräumliche Disparität und eine wachsende Polarisierung von Wohngebieten beobachten, also die Herausbildung von Gebieten mit hoher Problemakkumulation. In diesen Gebieten wurden vielerorts Projekte der Gemeinwesenarbeit umgesetzt, die sich unter maßgeblicher Beteiligung der Anwohner*innen und an den Interessen der Bewohner*innen orientiert für eine Verbesserung der Lebenssituation in den Gebieten einsetz(t)en.

Wurtzbacher (2014) konstatiert, dass von staatlicher Seite dieser Entwicklung der räumlichen Polarisierung seit Ende der 1990er-Jahre u. a. mit dem bundesweiten Städtebauförderprogramm Soziale Stadt begegnet wird, das als Topdown-Strategie konzipiert wurde und mithilfe integrierter Handlungskonzepte auf die Herausforderungen vor Ort reagiert (vgl. Wurtzbacher 2014: 270 ff.) Hierbei ist das Förderprogramm im Ressort Stadtentwicklung angesiedelt und der Förderschwerpunkt liegt auf baulichen Infrastrukturmaßnahmen. Der soziale Anteil des Programms ist in verschiedenen Bundesländern und Kommunen sehr verschieden gewichtet: In einigen Bundesländern ist er nicht existent (z. B. in Hessen und Niedersachsen) und in anderen Bundesländern wird damit Gemeinwesenarbeit, umbenannt in Quartiersmanagement, finanziert (Bremen). Bereits seit vielen Jahren fordert daher die Bundesarbeitsgemeinschaft Soziale Stadtentwicklung und Gemeinwesenarbeit e. V. (BAG) eine stärkere Betonung und Förderung sozialer Belange im Rahmen der Sozialen Stadtentwicklung durch die Einbettung und Einbeziehung von Gemeinwesenarbeit in allen Gebieten der Sozialen Stadt und darüber hinaus (vgl. BAG 2004: 1 ff.). 


\subsection{Arbeitsweise der Gemeinwesenarbeit}

Im Gegensatz zu anderen Handlungsfeldern der Sozialen Arbeit arbeitet die GWA grundsätzlich mit allen Personengruppen und Menschen vor Ort, setzt also gruppenübergreifend an. „Durch diese einzelfallübergreifende gruppen- und sozialraumbezogene Arbeit wird eine stigmatisierende Fokussierung auf einzelne Menschen oder Gruppen verhindert, gemeinsame Austausch- und Lernprozesse der Menschen untereinander werden befördert" (Riede et al. 2016: 4). Um die Lebenssituation mit den Bewohner*innen gestalten zu können, ist eine Verankerung der GWA in der Lebenswelt der Menschen im Stadtteil durch niedrigschwellige Anlaufpunkte in der Nachbarschaft erforderlich. Im Rahmen der Gemeinwesenarbeit werden die Menschen vor Ort als Expert*innen ihrer eigenen Lebenssituation betrachtet. Gemeinwesenarbeiter*innen agieren präventiv, proaktiv und problemlösend durch die rechtzeitige Stärkung der Ressourcen Einzelner, von Beziehungen oder materiellen und infrastrukturellen Bedingungen. Gleichzeitig suchen GWAMitarbeiter*innen Bündnispartner*innen für die Verbesserung der Lebensbedingungen auf allen Ebenen, auch in der Verwaltung und Politik (vgl. Lüttringhaus 2011: $277 \mathrm{f}$.).

Fehren (2016) betont diesbezüglich, dass es GWA um eine doppelte Aktivierungsrichtung von Bürger*innen und Institutionen geht und GWA als „Resonanzverstärker für Bürgeraktivitäten " in der Verwaltung fungiert. Gleichzeitig vermittelt GWA als intermediäre Instanz zwischen verschiedenen Personen, Institutionen und Akteursebenen mit je verschiedenen Handlungsrationalitäten und Sinnzusammenhängen (vgl. Fehren 2016: 62 ff.).

„Bei einer durch professionelle Soziale Arbeit und damit durch einen ,Systemakteur ausgeübten intermediären Funktion ist [jedoch] davon auszugehen, dass der intermediäre Balanceakt zwischen Lebenswelt und System, wenn er misslingt, tendenziell zuungunsten der Lebenswelt kippt“ (ebd.: 66).

Nach Einschätzung der Autorin lässt sich vielerorts beobachten, dass durch ein entsprechendes Management der Aktivitäten im Stadtteil und enge Zielvorgaben durch die Verwaltung (z. B. in vielen Gebieten der Sozialen Stadt) die Arbeit an der Basis mit den Menschen in den Hintergrund gedrängt bzw. vernachlässigt wurde.

Dabei wirft Stoik (2012) die Frage der Parteilichkeit der Gemeinwesenarbeiter*innen auf, also ob im Stadtteil alle einbezogen werden sollen oder die Schwächeren gezielt gestärkt werden müssen (vgl. Stoik 2012: 3). Diese Frage wurde im Laufe der Geschichte der GWA immer wieder kontrovers diskutiert und interpretiert. Angesichts der ethischen Grundlagen der Sozialen Arbeit und ihres Bekenntnisses zu Menschenrechten und sozialer Gerechtigkeit gilt es, ökonomisch und/oder 
sozial benachteiligte Menschen besonders einzubeziehen. Aber auch hinsichtlich der Wirksamkeit gilt es, Angebote passgenau zu gestalten und diejenigen mitgestalten zu lassen, die diese Angebote in Anspruch nehmen sollen.

\subsection{Verortung von Gemeinwesenarbeit}

Rothschuh und Spitzenberger (2010) verorten Gemeinwesenarbeit in der Schnittmenge aus Sozialer Stadtentwicklung, Sozialraumorientierung und Bürgerbeteiligung (siehe Abb. 1), wobei in Theorie und Praxis die Begrifflichkeiten verschwimmen, insbesondere zwischen Sozialer Stadtteilentwicklung und Sozialraumorientierung. Im Gegensatz zu zumeist punktuell stattfindenden Veranstaltungen der Bürgerbeteiligung ist GWA eine langfristig angelegte Beteiligungsund Ermutigungsarbeit. Gerade durch den langfristigen Vertrauensaufbau durch GWA-Mitarbeiter*innen im Stadtteil können auch Menschen gezielt angesprochen und eingebunden werden, die sich an herkömmlichen Beteiligungsverfahren nicht beteiligen würden.

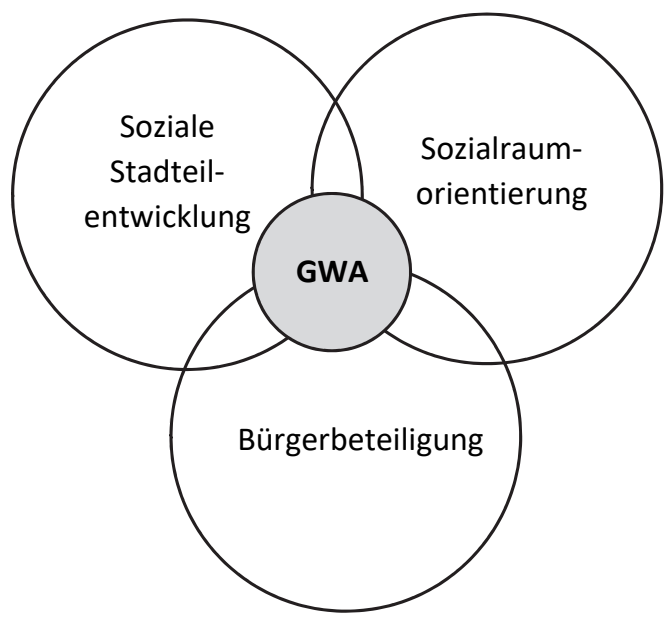

Abbildung 1: Kontexte der Gemeinwesenarbeit

Quelle: Rothschuh \& Spitzenberger (2010: 82). 


\subsection{Gemeinwesenarbeit als Methode, Arbeitsprinzip, Konzept etc.}

Während Gemeinwesenarbeit in der Vergangenheit sehr unterschiedlich charakterisiert und eingeordnet wurde, z. B. als Methode, Prozess, Strategie oder Arbeitsprinzip, wird zunehmend vom Konzept der Gemeinwesenarbeit gesprochen (vgl. Stövesand \& Stoik 2013: 17). Dieses Konzept bzw. seine verschiedenen Variationen fördern ,[...] die Entwicklung gemeinsamer Handlungsfähigkeit und kollektives Empowerment bezüglich der Gestaltung bzw. Veränderung von infrastrukturellen, politischen und sozialen Lebensbedingungen [...]" (Stövesand \& Stoik 2013: 16). Diese Veränderung hin zum Konzept wird damit begründet, dass vorhandene GWA-Ansätze in der Regel Ziele, Erklärungen, Begründungen, Methoden und Techniken verknüpfen und damit den Anforderungen an ein Konzept entsprechen. Allerdings handelt es sich bei GWA ,[...] um ein Konzept, das in unterschiedlichen Varianten existiert, vermittelt und umgesetzt ist" (Stövesand 2018: 4). Im Laufe der Geschichte haben diese GWA-Varianten verschiedene Ausprägungen und unterschiedliche Formen angenommen. Oelschlägel (2017) ist der Ansicht: „GWA bildet immer ein Kontinuum, an dessen einem Ende ein systemkritischer, konfliktorientierter, zu Zeiten auch sozialrevolutionärer Ansatz steht und am anderen Ende ein staatstragender, systemorientierter meist pragmatisch-managerieller Ansatz" (Oelschlägel 2017: 172). Hier wird deutlich, dass es sehr verschieden ausgerichtete GWA-Projekte gab und gibt, die zumeist stark von gesellschaftlichen Entwicklungen und dem entsprechenden Zeitgeist geprägt wurden bzw. werden. An dieser Stelle sei auch der besonders basisnahe Strang der GWA erwähnt, das Community Organizing, das Organisationen, Gruppen und Institutionen im Stadtteil als organisierte Zivilgesellschaft zusammenbringt. Diese handlungsfähigen Bürgerplattformen, in denen Menschen unterschiedlichster Herkunft, Weltanschauung und Schicht zusammenkommen, praktizieren eine basisbezogene, demokratische Teilhabe. Nachdem sie eine gemeinsame Beziehungsebene aufgebaut haben, wählen sie gemeinsam Themen im Stadtteil aus, nehmen die politische Gesellschaft in die Pflicht und setzten sich für positive Veränderungen ein (vgl. Penta 2007: 9).

\subsection{Herausforderungen für die Gemeinwesenarbeit}

Die Ursachen dafür, dass die Gemeinwesenarbeit viele Jahre ein Nischendasein geführt hat und noch immer führt, sind vielfältig. Hierzu zählen u. a.:

- $\quad$ keine kongruente Verwendung des Begriffs Gemeinwesenarbeit sowie die Einführung vielfältiger neuer Bezeichnungen für die Soziale Arbeit im Gemeinwesen, 
- die Ausbreitung der Idee der Gemeinwesenarbeit in einzelfallbezogene Handlungsfelder der Sozialen Arbeit als Fachkonzept Sozialraumorientierung,

- das Aufgreifen der Sozialraumorientierung auch in der Stadtentwicklung und die Einführung des bundesweiten Stadtentwicklungsprogramms Soziale Stadt mit Quartiersmanagement-Teams, vielerorts mit nur geringer Beteiligung sozialer Akteur*innen,

- die Einführung einer intermediären Handlungsebene und zunehmende Verlagerung der Arbeit von der Basis in Stadtteilbüros auf die intermediäre, vermittelnde und managende Handlungsebene (vgl. Riede 2016: 327),

- eine einzelfallbezogene, zielgruppenspezifische Finanzierung von Hilfen in der Sozialen Arbeit auf die Rechtsansprüche bestehen, während die Förderung von Nachbarschaftsarbeit keine Pflichtaufgabe ist und sich Nachbarschaftseinrichtungen zumeist aus verschiedenen projektbezogenen Fördertöpfen finanzieren müssen,

- wenig (interdisziplinäre) Kooperation zwischen Theorie- und Praxisnetzwerken im Bereich Gemeinwesenarbeit und Soziale Stadtentwicklung.

Trotz dieser Entwicklungen haben sich Stadtteil- und Nachbarschaftszentren in Deutschland an vielen Orten etabliert und über Jahre hinweg gemeinwesenorientiert gearbeitet, parallel zum Programm Soziale Stadt und dem Quartiersmanagement. Allerdings haben diese Nachbarschaftseinrichtungen zumeist mit prekärer Finanzierung zu kämpfen, sodass sich die Arbeit teilweise auf soziale und kulturelle Angebote beschränkt; strukturelle Veränderungen, wie konzeptionell vorgesehen, sind damit selten realisierbar. GWA gründet sich auf historische Erfahrungen und wird getragen von der gesellschaftlichen Vision eines lebendigen, inklusiven und gerechten Miteinanders in Nachbarschaften. Hierbei ist eine vertikale sowie horizontale Vernetzung der Menschen, Akteur*innen und Ebenen im Gemeinwesen wichtig. Aushandlungsprozesse zwischen verschiedenen Interessen sind erforderlich und hier liegt die Schnittstelle zur lokalen Demokratieentwicklung, die bislang unzureichend erforscht ist.

\section{Gemeinwesenarbeit als demokratiefördernde Brückenbauerin - konzeptionelle Grundlagen}

Damit in heterogener werdenden Nachbarschaften ein gutes Miteinander entstehen kann und Teilhabemöglichkeiten für alle Bewohner*innen geschaffen werden können, werden Akteur*innen benötigt, die langfristig, nachhaltig und präventiv alle in den Blick nehmen und den Stadtteil gemeinsam mit den Bewohner*innen in Richtung einer positiven Zukunftsentwicklung begleiten. Im Sinne einer Teilhabe für alle und angesichts aktueller gesellschaftlicher Herausforderungen gilt es, 
die konzeptionellen Aspekte der Gemeinwesenarbeit zu einem innovativen, demokratiefördernden, nachhaltigen und inklusiven Ansatz im Gemeinwesen weiterzuentwickeln. Hierbei erscheinen die folgenden drei theoretisch-konzeptionellen Ansätze bedeutsam, die aus Tabelle 1 hervorgehen:

Tabelle 1: Theoretisch-konzeptionelle Bezüge von Gemeinwesenarbeit als Brückenbauerin

\begin{tabular}{l|l|l}
\hline \multicolumn{1}{l}{ Theoretisch-konzeptionelle Bezüge } & \multicolumn{2}{l}{ Inhalt } \\
\hline Prinzipien & Konzepte & $\begin{array}{l}\text { Soziales, insbesondere } \\
\text { brückenbildendes Kapital } \\
\text { ausbauen - zielgruppen- } \\
\text { übergreifendes Miteinan- } \\
\text { Miteinanders } \\
\text { der fördern }\end{array}$ \\
\hline $\begin{array}{l}\text { Verbesserung des kom- } \\
\text { munikativen Austauschs } \\
\text { und der Konfliktvermitt- } \\
\text { lung }\end{array}$ & $\begin{array}{l}\text { Deliberdieu 1992; Putnam } \\
\text { 1998) und emanzipatori- } \\
\text { sches (Alcántara et al. } \\
\text { 2014) Demokratiemodell }\end{array}$ & $\begin{array}{l}\text { Teilhabemöglichkeiten } \\
\text { für alle Anwohner*innen } \\
\text { an öffentlichen Beteili- } \\
\text { gungs- und Kommunika- } \\
\text { tionssettings zur Verbes- } \\
\text { serung des kommunikati- } \\
\text { ven Austauschs und der } \\
\text { Konfliktvermittlung }\end{array}$ \\
\hline $\begin{array}{l}\text { Entwicklung einer nach- } \\
\text { haltigen Gesellschaftsper- } \\
\text { spektive }\end{array}$ & $\begin{array}{l}\text { Gemeinwesenökonomie } \\
\text { (Elsen 2011) }\end{array}$ & $\begin{array}{l}\text { Nachhaltige Gesell- } \\
\text { schaftsentwicklung an- } \\
\text { streben - gemeinsame } \\
\text { Visionssuche und Ausbau } \\
\text { der Gemeinwesenökono- } \\
\text { mie }\end{array}$ \\
\hline
\end{tabular}

Quelle: Eigene Darstellung

\subsection{Förderung des sozialen Miteinanders}

Bei der Betrachtung der Beziehungen zwischen Akteur*innen und der sich daraus ergebenden Konsequenzen ist der Bezug auf die Theorie des Sozialen Kapitals (vgl. Bourdieu 1992: 49 ff.) naheliegend. Einer gemeinwesenbezogenen Arbeit geht es um gemeinschaftliche Zusammenhänge. Daher stehen soziale Beziehungen und Netzwerke zwischen Personen und deren kollektive Aktionen im Vordergrund. Dieser Fokus auf Netzwerke und ihre Ressourcen lenkt den Blick auf die 
theoretische Kategorie des Sozialen Kapitals. Analytisch wird zwischen bindenden (bonding), brückenbildenden (bridging) und institutionenbezogenen (linking) Beziehungen unterschieden (vgl. u. a. Putnam 2000; Karstedt 2004). Während also bindende Beziehungen zwischen ähnlichen Personen entstehen, verknüpfen brückenbildende Beziehungen verschiedene Akteursgruppen miteinander, wohingegen institutionenbezogene Beziehungen auf Kontakte zwischen Menschen und Institutionen verweisen. Durch die Aktivierung der Menschen im Rahmen der Gemeinwesenarbeit sollen Vorteile des Sozialkapitals erzeugt werden, wobei ein Bewusstwerden gemeinsamer Interessen angestrebt wird, also eine Solidarisierung der Bewohner*innen. Während bindendes Sozialkapital dabei hilft, im täglichen Leben zurechtzukommen, kann das brückenbildende Sozialkapital eher beim Aufstieg behilflich sein, z. B. bei der Arbeits- oder Wohnungssuche. Professionell handelnde Gemeinwesenarbeiter*innen können hier als Verbindungskapital aktiv werden (vgl. Landhäußer 2013: 154 ff.). Putnam (1993) konstatiert: „Building social capital will not be easy, but it is the key to making democracy work" (Putnam 1993: 185).

Da im Gemeinwesen verschiedene Gruppen nebeneinander existieren, die oft wenig Kontakt zueinander haben, erscheinen Möglichkeiten zum Austausch und Brückenbau zwischen den Gruppen interessant. Dies erfordert ein ressourcenorientiertes und vernetzendes Vorgehen der Gemeinwesenarbeiter*innen. Von zentraler Bedeutung ist, dass ausreichend Zeit für die Arbeit im Stadtteil, für Gespräche mit den Menschen und Gruppen vor Ort, also für eine „Innenansicht“ des Stadtteils und den Aufbau von Vertrauen, vorhanden ist. Beziehungsarbeit zur Ermutigung und Befähigung von Menschen benötigt Zuwendung und Zeit. Bei den Gesprächen mit verschiedenen Menschen gilt es, Interessen und unterschiedliche Bedürfnisse zu verstehen und gemeinsame Themen herauszufiltern. Dazu bedarf es u. a. einer Sprache und Ausdrucksweise, die von der jeweiligen Zielgruppe verstanden wird und die gesprächsfördernd ist. Ähnliche Interessen von Menschen sollen milieuübergreifend zusammengeführt und die Beteiligten im Sinne von Hilfe zur Selbsthilfe bei diesen kollektiven Selbstorganisationsprozessen unterstützt werden. Hierbei gilt es auch, verstärkt migrantische Selbstorganisation zu fördern und einzubeziehen (zu Forschungsansätzen zu Sozialkapital und Quartiersentwicklung siehe Drilling \& Schnur 2012: 20).

Der Arbeits- und Handlungsansatz der Gemeinwesenarbeit, der an den Interessen und Bedürfnissen der Menschen vor Ort ansetzt, fördert eine demokratische Zivilgesellschaft unter Einbeziehung aller Menschen vor Ort. „Ziel der Gemeinwesenarbeit ist es, mit Hilfe von Ressourcenaktivierung, partizipativer Projektarbeit und Empowerment Menschen und Akteursgruppen im Sozialraum zur aktiven Mitgestaltung ihres Gemeinwesens anzuregen. Dabei werden zivilgesellschaftliche, menschenrechtsorientierte Strukturen geschaffen und lokale Strategien zur 
Etablierung dieser entwickelt" (Bringt et al 2014: 13). Von besonderer Bedeutung ist hierbei, dass vorhandenes bürgerschaftliches Engagement je nach Bedarf der Engagierten, z. B. durch Beratung, räumliche und sonstige Angebote, unterstützt wird. „Der Begriff grassroots (englisch: Graswurzeln) [...] wird mit Aktivitäten von der Basis, von den Bürgerinnen und Bürgern, mit Basisdemokratie oder auch sozialen Bewegungen assoziiert" (Voss 2010). Diese basisdemokratischen Aktivitäten unterstützt Gemeinwesenarbeit lokal vor Ort. Aber darüber hinaus zielt Gemeinwesenarbeit insbesondere auch darauf ab, die Interessen und Bedürfnisse von ökonomisch und/oder sozial benachteiligten Menschen einzubeziehen, diese Menschen zu befähigen und zu ermutigen. Durch vielfältige kleinere und größere Teilhabe-, Partizipations- und Bildungsangebote soll allen Menschen die Teilnahme an demokratischen Beteiligungsprozessen ermöglicht und ein Miteinander angeboten werden. Es gilt, die Ideen und Bedürfnisse von Menschen vor Ort durch Gespräche herauszufiltern und diese bei der Umsetzung im Sinne eines kollektiven Empowerments zu unterstützen. Da alle Menschen über unterschiedliche Potenziale und Kompetenzen verfügen, sollen diese ressourcenorientiert einbezogen und ausgetauscht werden.

\subsection{Verbesserung des kommunikativen Austauschs und der Konfliktvermittlung}

Die Förderung einer lebendigen Dialog- und Partizipationskultur im Stadtteil sowie die Vermittlung zwischen unterschiedlichen und z. T. gegenläufigen Interessen sind zentrale gesellschaftliche Aufgaben. Selbstverständlich haben Menschen im Stadtteil unterschiedliche Meinungen und Interessen und die Auseinandersetzung mit den verschiedenen Interessen ist häufig nicht konfliktfrei, sondern erzeugt Reibung. Hier werden Gemeinwesenarbeiter*innen als Mittler*innen, Moderator*innen und Mediator*innen tätig und vermitteln zwischen verschiedenen Interessen im Stadtteil.

Veränderungen (vor Ort) verunsichern und wecken das Bedürfnis über sie zu sprechen. Doch wo kommen die Menschen selbst zu Wort und können sich über die stattfindenden Veränderungen austauschen? Nur in wenigen Nachbarschaften oder Stadtteilen gibt es eine institutionalisierte lokale Diskussionskultur jenseits von Parteipolitik. Mit dem deliberativen Modell der Zivilgesellschaft betont Habermas (1998) die Bedeutung dessen, auch informelle oder wenig formell gebildete öffentliche Meinungen aus der Lebenswelt der Menschen in das politischadministrative System einzuspeisen. Diesem Verständnis entsprechend erfolgt Integration in die Gesellschaft nicht durch geteilte Werte, sondern mittels effektiver Beteiligungsmöglichkeiten der Bürger*innen an der demokratischen Kontrolle. Habermas' zivilgesellschaftsbezogenes Interesse gilt hierbei insbesondere ihrer demokratiefördernden und demokratiestabilisierenden Funktion; die deliberative 
Zivilgesellschaft wird als Instanz politisch-gesellschaftlicher Risikobewertung gesehen (vgl. Fehren 2006: 58 f.). Aber auch um die Potenziale und Fähigkeiten der Stadtteilbewohner*innen sichtbar zu machen, können offene Dialoge in Form von quartiersöffentlichen Bürgerforen durchgeführt werden. Hierdurch werden Anliegen und Schwerpunktsetzungen generiert, die dann in die Struktur geförderter Projekte, das Verwaltungshandeln sowie Möglichkeiten zur Selbsthilfe einfließen (vgl. Wurtzbacher 2014: 278).

Im Sinne eines emanzipatorischen Demokratiemodells (Alcántara et al. 2014) gilt es, auf die Inklusion und das Empowerment aller Menschen und Akteur*innen bei partizipativen Verfahren im Stadtteil zu achten. Durch vielfältige kleinere und größere Dialogveranstaltungen und Partizipationsmöglichkeiten, von der klassischen Dialogveranstaltung am Abend bis hin zu Gesprächsrunden an Spielplätzen oder Straßenfesten, gilt es, verschiedene Formate zu kreieren, um möglichst vielen verschiedenen Menschen die Teilnahme zu ermöglichen. Gemeinwesenarbeit fördert die Auseinandersetzung mit verschiedenen Meinungen, die Konfliktvermittlung und die Suche nach Win-win-Lösungen.

Die Arbeitsweise der Gemeinwesenarbeit fördert ein demokratisches Miteinander auf lokaler Ebene. Gerade auch durch die Gestaltung von Partizipationsmöglichkeiten an Entscheidungen wird die aktive Teilhabe an der Zivilgesellschaft für die Menschen vor Ort zur erlebbaren Realität (vgl. Riede et al. 2016: 3). An für alle zugänglichen und „,neutralen“ Orten werden Begegnungs- und Austauschmöglichkeiten für alle Menschen verschiedener Milieus angeboten. „Insbesondere in einer Gesellschaft, die durch soziale Spaltung, marginalisierte Bevölkerungsgruppen und eine Ökonomisierung des Sozialen gekennzeichnet ist, stellt die Gemeinwesenarbeit einen unverzichtbaren Bestandteil der Sozialen Arbeit dar, um das Interesse aller Bewohner*innen am öffentlich-politischen Leben aufrechtzuerhalten, an einer Demokratisierung der Gesellschaft weiterzuarbeiten und gegen Politikverdrossenheit aktiv vorzugehen" (Aschenbrenner-Wellmann \& Ehrismann 2014: 370). Rausch (2017) betont, dass Gemeinwesenarbeit im Selbstverständnis von Profession und Disziplin der Sozialen Arbeit geradezu prädestiniert dazu ist, gegen den zunehmenden (Rechts-)Populismus wirksame Beiträge zu leisten. Allerdings können die Erwartungen angesichts fehlender Forschungsprojekte und valider Evaluationen bislang nicht hinreichend gesichert werden (vgl. Rausch 2017: 4 und auch seinen Beitrag in diesem Band).

\subsection{Entwicklung einer nachhaltigen Gesellschaftsperspektive}

Angesichts des gesellschaftlichen Leitbildes einer nachhaltigen Entwicklung in Deutschland und zunehmender klimatischer Veränderungen gilt es, eine nachhaltige Gesellschaftsentwicklung verstärkt auch lokal vor Ort in den Blick zu nehmen und 
diesbezügliche Aktivitäten auf die Agenda zu setzen. Drilling und Schnur (2012) stellen fest: „Quartiere können als Interventionsebene, mehr aber vielleicht noch als Nukleus nachhaltiger Stadtentwicklungsprozesse verstanden werden. Dabei sind die drei Nachhaltigkeitsdimensionen ,ökologisch', ,ökonomisch' und ,sozial' im Auge zu behalten“ (Drilling \& Schnur 2012: 17). Der Komplexität einer globalisierten Ökonomie kann durch konkrete, nachhaltige Projekte, die den Menschen Selbstwirksamkeitserfahrungen ermöglichen und ihnen dadurch Mut und Hoffnung machen, lokal vor Ort begegnet werden. Gemeinsam gilt es, eine zukunftsfähige lokale Entwicklung vorauszudenken, wobei die Gemeinwesenarbeit langjährige Erfahrung in der Anwendung zukunftsorientierter Methoden wie z. B. der Aktivierenden Befragung und der Zukunftswerkstatt hat. Allerdings konstatieren Drilling et al. (2015), dass die utopischen Elemente von Gemeinwesenarbeit im Zuge der Entwicklung zum Fachkonzept Sozialraumorientierung zur Reorganisation Sozialer Arbeit verdrängt worden seien (vgl. Drilling et al. 2015: 31). Die Entwicklung gemeinsamer Ideen und Ziele für die Zukunft, eine gemeinsame Visionssuche, kann jedoch ggf. dabei helfen, aktuelle Spannungen oder Konflikte zu überwinden.

Interessant erscheint in diesem Kontext auch die Diskussion um die Gemeinwesenökonomie, die in den 1990er-Jahren in Deutschland angesichts hoher Arbeitslosenquoten intensiver diskutiert wurde. Gemeinwesenökonomie wird häufig auch mit Solidarischer Ökonomie, Alternativer Ökonomie oder Lokaler Ökonomie gleichgesetzt (vgl. Notz 2012: 123).

„Lokale und regionale Lebensräume ermöglichen die umfassende Gestaltung in all den Wirkungsbereichen, die örtlich verankert sind. Der Raumbezug hebt die Trennung der sozialen, ökologischen und ökonomischen Sphären potentiell auf und ermöglicht integrierte Handlungsansätze sowie Lernen für eine ökosoziale Zukunft“ (Elsen 2011: 99).

Oelschlägel (2013) bezeichnet GWA und lokale Ökonomie als einen Strang der neueren Entwicklungen der Gemeinwesenarbeit. Menschen, die aus dem Arbeitsprozess herausfallen und auf ihr Lebensumfeld als zusätzliche Ressource zur Existenzsicherung angewiesen sind, könnten mit dem wachsenden Bedarf in benachteiligten Quartieren bei der Infrastrukturgestaltung und den sozialen Dienstleistungen in quartiersbezogenen basisökonomischen Projekten zusammengeführt werden. So würden im Sinne der Gemeinwesenökonomie nichtprofitorientierte Strategien der Selbsthilfe und Selbstorganisation gebündelt (vgl. Oelschlägel 2013: $194 \mathrm{f}$.).

$\mathrm{Zu}$ den aktuellen Konzepten der Gemeinwesenökonomie zählt Notz auch die Tauschökonomie, die Schenkökonomie und die Umsonstökonomie, selbstverwaltete Dorfläden und Guerilla Gardening/Gemeinschaftsgärten (vgl. Notz 2012: 124 ff.). Einige Studien belegen jedoch, dass Ansätze der Gemeinwesenökonomie 
insbesondere von Personen mit höherer Bildung und hohem gesellschaftspolitischen Engagement genutzt werden (vgl. Wagner 2009: $148 \mathrm{f}$.). Hier stellt sich die Frage, wie diese Ansätze weiterentwickelt werden können, sodass sie auch für einen erweiterten Personenkreis interessant und zugänglich werden. Hinsichtlich der strukturellen Förderung von solidarischer Ökonomie lohnt sich ein Blick in Richtung Italien, Kanada und Brasilien. Schnee (2009) ist der Ansicht:

„Kooperatives Wirtschaften braucht die Gestaltung zwischenmenschlicher Interaktionen zur Realisierung gemeinschaftlich angestrebter wirtschaftlicher Ziele - eine Kernaufgabe der GWA. Für kleine Projekte im Stadtteil braucht es vor allem Fantasie und Durchhaltevermögen, für große braucht es viele weitere Kontakte und die Vision ,Es ist möglich!““ (Schnee 2009).

\section{Gemeinwesenarbeit als Brückenbauerin - Prinzipien}

Im Rahmen der Gemeinwesenarbeit geht es um die Hilfe und Befähigung der Menschen zur Selbsthilfe, um Kooperation und Vernetzung sowie um die Nutzung und Ermöglichung neuer Beziehungen im Stadtteil, also um den Ausbau des sozialen Kapitals. Für die Gemeinwesenarbeit wurden Leitstandards entwickelt (Lüttringhaus 2011), die von Riede, Rothschuh, Stracke-Baumann und Zychlinski (2017) weiterentwickelt wurden, um die sozialen Stärken der GWA als Brückenbauerin weiter zu konkretisieren (siehe Tab. 2). Dies erscheint insbesondere hinsichtlich der häufig verkürzten, unpolitischen Rezeption von GWA sinnvoll (siehe Kapitel 5, vgl. Riede et al. 2017; aufbauend auf Lüttringhaus 2011: 277).

Tabelle 2: Prinzipien von Gemeinwesenarbeit als Brückenbauerin

\begin{tabular}{l|l}
\hline $\begin{array}{l}\text { Gruppenüber- } \\
\text { greifendes Han- } \\
\text { deln - Vielfalt } \\
\text { erlebbar ma- } \\
\text { chen }\end{array}$ & $\begin{array}{l}\text { Gemeinwesenarbeit hat alle Menschen im Sozialraum im Blick } \\
\text { und bringt verschiedene Personen und Gruppen miteinander in } \\
\text { Kontakt. Gleichzeitig wird auch mit verschiedenen Personen- } \\
\text { gruppen gezielt gearbeitet, um diese besonders zu befähigen } \\
\text { und zu unterstützen, z. B. Familien, Frauen, Arbeitslose, ge- } \\
\text { flüchtete Frauen, Senior*innen. Darüber hinaus werden the- } \\
\text { menbezogene Gruppen begleitet (z. B. zu den Themen Woh- } \\
\text { nen, Verkehr, Freizeitgestaltung, Grünflächenverbesserung), } \\
\text { bei denen verschiedene Menschen interessenbezogen zusam- } \\
\text { menfinden. Begegnungsmöglichkeiten für alle Menschen im } \\
\text { Stadtteil werden geschaffen, z. B. Nachbarschaftscafés, und } \\
\text { durch gemeinsame Aktionen, Feste etc. wird ein wechselseiti- } \\
\text { ger Ressourcenaustausch und ein voneinander Lernen ermög- } \\
\text { licht. }\end{array}$ \\
\hline
\end{tabular}




\begin{tabular}{|c|c|c|}
\hline 2. & $\begin{array}{l}\text { Orientierung an } \\
\text { den Bedürfnis- } \\
\text { sen und The- } \\
\text { men der Men- } \\
\text { schen }\end{array}$ & $\begin{array}{l}\text { Gemeinwesenarbeit setzt am Willen, den Bedürfnissen und In- } \\
\text { teressen der Menschen an. Hierbei sind die Bedürfnisse und In- } \\
\text { teressen der Menschen vor Ort Ansatzpunkte für Veränderun- } \\
\text { gen in der Gestaltung des Alltags. }\end{array}$ \\
\hline 3. & $\begin{array}{l}\text { Kommunika- } \\
\text { tive Vermitt- } \\
\text { lung zwischen } \\
\text { unterschiedli- } \\
\text { chen Lebens- } \\
\text { welten }\end{array}$ & $\begin{array}{l}\text { Als Mittlerin zwischen den Menschen, ihren verschiedenen In- } \\
\text { teressen und Bedürfnissen fördert die Gemeinwesenarbeit die } \\
\text { Kommunikation über Veränderungsprozesse und den Umgang } \\
\text { mit zunehmender Diversität. Damit trotz Verschiedenheit Vor- } \\
\text { urteile abgebaut werden können und Vertrauen wachsen kann, } \\
\text { braucht es Möglichkeiten der Begegnung sowie positive Erfah- } \\
\text { rungen im Umgang miteinander. Durch verschiedene Dia- } \\
\text { logsettings und die Ermöglichung einer offenen, lösungsorien- } \\
\text { tierten Kommunikation fördert die Gemeinwesenarbeit Aus- } \\
\text { tauschprozesse im Sozialraum. }\end{array}$ \\
\hline 4. & $\begin{array}{l}\text { Förderung von } \\
\text { kollektivem } \\
\text { Empowerment } \\
\text { und Selbstorga- } \\
\text { nisation }\end{array}$ & $\begin{array}{l}\text { Allen Menschen stehen Anerkennung, Respekt, Selbstwirk- } \\
\text { samkeitserfahrungen und Hilfe zur Selbsthilfe zu. Gemeinwe- } \\
\text { senarbeit führt ähnliche Interessen von Menschen zusammen, } \\
\text { unterstützt und ermutigt die Menschen zur Selbstorganisation, } \\
\text { wodurch zivilgesellschaftliche Potenziale gestärkt und Syner- } \\
\text { gieeffekte erzeugt werden. Ein besonderer Fokus wird auf das } \\
\text { kollektive Empowerment (die Ermutigung, Befähigung und Er- } \\
\text { mächtigung) der ökonomisch und/oder sozial besonders be- } \\
\text { nachteiligten Menschen gelegt, um ihnen mehr Teilhabe, Mit- } \\
\text { sprache und Mitgestaltung zu ermöglichen. }\end{array}$ \\
\hline 5. & $\begin{array}{l}\text { Partizipative } \\
\text { (Bildungs-)Mög- } \\
\text { lichkeiten } \\
\text { schaffen }\end{array}$ & $\begin{array}{l}\text { GWA schafft Möglichkeitsräume für Partizipation im Gemein- } \\
\text { wesen und für die Erfahrungen von Selbstwirksamkeit. Dies } \\
\text { kann auf vielfältige, kreative Weise geschehen, durch kleine } \\
\text { und große partizipative Beteiligungsmöglichkeiten. Durch An- } \\
\text { gebote für solidarische Aktionen und Begegnungen wird die } \\
\text { Entwicklung von Respekt und gegenseitigem Verständnis zwi- } \\
\text { schen verschiedenen Menschen und Gruppen gefördert. Partizi- } \\
\text { pative Ansätze sind hierbei als Lernsettings zu sehen, in denen } \\
\text { die Menschen demokratisches Miteinander erlernen. }\end{array}$ \\
\hline 6. & $\begin{array}{l}\text { Nutzung der } \\
\text { vorhandenen } \\
\text { Ressourcen }\end{array}$ & $\begin{array}{l}\text { Gemeinwesenarbeiter*innen unterstützen Menschen dabei, sich } \\
\text { ihrer individuellen Fähigkeiten, Kompetenzen und Ressourcen } \\
\text { bewusst zu werden. Darüber hinaus werden Ressourcen von In- } \\
\text { dividuen und kollektiven Akteur*innen gebündelt bzw. mitei- } \\
\text { nander vernetzt und auch institutionelle Ressourcen im Sozial- } \\
\text { raum bestmöglich gemeinschaftlich genutzt. }\end{array}$ \\
\hline
\end{tabular}




\begin{tabular}{|c|c|c|}
\hline 7. & $\begin{array}{l}\text { Ressortüber- } \\
\text { greifendes } \\
\text { Handeln }\end{array}$ & $\begin{array}{l}\text { Zur Realisierung und Bewältigung der umfassenden Ziele der } \\
\text { Gemeinwesenarbeit ist eine interdisziplinäre und fach- bzw. } \\
\text { ressortübergreifende Zusammenarbeit erforderlich. GWA } \\
\text { agiert hier als intermediäre Mittlerin zwischen Anwohner*in- } \\
\text { nen, Politik und Verwaltung. }\end{array}$ \\
\hline 8. & $\begin{array}{l}\text { Vernetzung und } \\
\text { Kooperation }\end{array}$ & $\begin{array}{l}\text { Die verschiedenen Bewohner*innen und Akteur*innen im So- } \\
\text { zialraum gilt es, miteinander zu vernetzen; (projektbezogene) } \\
\text { Kooperationen sollen umgesetzt werden. Dadurch können im } \\
\text { Sinne einer positiven Zukunftsentwicklung Doppelstrukturen } \\
\text { abgebaut, Synergieeffekte erzeugt und eine effektive Zusam- } \\
\text { menarbeit erreicht werden. Weiterhin gilt es, ehrenamtliches } \\
\text { Engagement strukturiert in die Arbeit im Stadtteil einzubezie- } \\
\text { hen, entsprechende Vernetzungsrunden (aus traditionellem und } \\
\text { neuem Ehrenamt) zu koordinieren und einen gegenseitigen Er- } \\
\text { fahrungsaustausch zu unterstützen. }\end{array}$ \\
\hline 9. & $\begin{array}{l}\text { Komm- und } \\
\text { Gehstruktur }\end{array}$ & $\begin{array}{l}\text { Gemeinwesenarbeit zeichnet sich einerseits dadurch aus, dass } \\
\text { es einen Ort der Begegnung gibt (Nachbarschaftstreff, Stadt- } \\
\text { teilzentrum), zu dem die Menschen kommen können. Anderer- } \\
\text { seits sind Gemeinwesenarbeiter*innen auch als Akteur*innen } \\
\text { im Stadtteil unterwegs und sprechen mit Menschen, die nicht } \\
\text { den Weg ins Stadtteilzentrum finden. So können weitere aktu- } \\
\text { elle Themen und Probleme der Menschen erkannt und frühzei- } \\
\text { tig aufgegriffen werden. }\end{array}$ \\
\hline 10. & $\begin{array}{l}\text { Nachhaltige } \\
\text { Gesellschafts- } \\
\text { entwicklung } \\
\text { im Blick }\end{array}$ & $\begin{array}{l}\text { Im Rahmen der Arbeit im Gemeinwesen behalten die GWA- } \\
\text { Mitarbeiter*innen den Stadtteil auch immer hinsichtlich seiner } \\
\text { sozialen, ökologischen und ökonomischen Entwicklungen im } \\
\text { Blick. Vor Ort werden Maßnahmen und Projekte gefördert, die } \\
\text { im Sinne einer nachhaltigen Entwicklung eine langfristige, so- } \\
\text { zial gerechte Entwicklung des Sozialraums befördern, ein um- } \\
\text { weltverträgliches Miteinander im Blick haben und eine nach- } \\
\text { haltige Lokale Ökonomie unterstützen. }\end{array}$ \\
\hline
\end{tabular}

Quelle: eigene Darstellung; aufbauend auf Riede et al. 2017

\section{Gemeinwesenarbeit zwischen Grassroots-Bewegungen und Fremdbestimmung}

GWA arbeitet mit Bürgerinitiativen und Graswurzelbewegungen zusammen, wo sie vorhanden sind. So manches GWA-Projekt wurde durch den Einsatz zivilgesellschaftlicher Akteur*innen erst ermöglicht, die finanziellen Mittel von diesen erstritten. Mancherorts werden ausschließlich von ehrenamtlich Engagierten 
beeindruckende Verbesserungen realisiert. Gleichzeitig besteht aber eine gewisse Gefahr der Vereinnahmung bzw. der einseitigen Vorteilsnahme; außerdem sind die Projekte häufig nicht von langer Dauer.

Selbstorganisationsprozesse sind voraussetzungsvoll und viele Menschen kommen nicht auf die Idee, haben keine Kapazitäten oder zeigen kein Interesse dafür, sich für die Verbesserung ihres eigenen Lebensumfeldes zu engagieren. Professionelle GWA Mitarbeiter*innen ohne Eigeninteressen im Stadtteil können zwischen verschiedenen Interessengruppen vermitteln, demokratische Grundlagen praktisch vermitteln und auch besonders die Teilhabe und Selbstwirksamkeitserfahrungen von Menschen in schwierigen Lebenssituationen in den Blick nehmen. Professionelle GWA verfügt über methodische Zugänge und Prinzipien, die - so sie partizipativ eingesetzt werden - im Austausch mit den Beteiligten passgenaue Dialogformen und Angebote schaffen. Eine Vielzahl neuer, verschiedener Bürgerbeteiligungsformate sind in den vergangenen Jahrzehnten (weiter)entwickelt worden und mancherorts konnten gute Ergebnisse erzielt werden. Auch neue intermediär aktive Stadtentwicklungsinitiativen sind an verschiedenen Stellen im Einsatz (vgl. Beck et al. 2017). Wenngleich gerade mit konkreten Beteiligungsformaten Menschen in ihrer Nachbarschaft häufig gut erreicht und einbezogen werden können, so ist doch die Einbindung kleinteiliger Formate in eine langfristige Strategie für den Stadtteil und insbesondere die Anbindung an kontinuierliche Ansprechpartner*innen wichtig, um u. a. durch einen Beziehungs- und Vertrauensaufbau eine Beteiligung heterogener Bevölkerungsmilieus zu ermöglichen.

Weiterhin muss in Bezug auf die verschiedenen Bürgerbeteiligungsveranstaltungen die Frage nach der Unabhängigkeit bzw. Offenheit von Auftraggeber*innen für neue Ergebnisse gestellt werden. Es zeigt sich, dass es bei Beteiligungsverfahren auch immer wieder um eine „Akzeptanzbeschaffung“ für kommunale, staatliche oder unternehmerische Projekte geht, die als Beispiele für eine Fremdbestimmung durch Scheinbeteiligung angesehen werden können. Das Thema der Fremdbestimmung ist für die Gemeinwesenarbeit in verschiedener Form immer wieder aktuell. So kann die politische Rahmensetzung, mit der sich die GWA auseinandersetzen muss, als Fremdbestimmung erlebt werden, da die Gemeinwesenarbeit sich immer wieder an unterschiedliche Förderprogramme und deren Förderund Berichtslogik anpassen und den Fokus ihrer Arbeit damit verändern muss. Im Kontext des Städtebauförderprogramms Soziale Stadt und auch bei anderen Förderprogrammen gibt es z. T. enge Zielvorgaben von Politik oder Verwaltung für die zu leistende Arbeit, die aufgrund statistischer Daten als Problemlagen identifiziert wurden. Diese Ziele decken sich ggf. nicht mit den Interessen und der Selbstmotivation der Menschen vor Ort oder beschränken die Quartiersmanager (stark) in ihrer inhaltlichen Arbeit, können also auch als Fremdbestimmung wahrgenommen werden. 
Der methodische Arbeitsansatz der Gemeinwesenarbeit ist dagegen ein anderer: Durch aktivierende Befragungen wird die Stimmung im Gebiet erfasst und werden Problemlagen aus Sicht der vor Ort lebenden Menschen identifiziert. Anschließend wird versucht, die Menschen zur Verbesserung der Lebenssituation zu aktivieren. In Gebieten der Sozialen Stadt und darüber hinaus gilt es also, übergeordnete Ziele der Verwaltung und die Interessen der Menschen vor Ort auszuhandeln, nach Möglichkeit in Einklang zu bringen und gemeinschaftlich an der Gebietsentwicklung zu arbeiten. Weiterhin lässt sich eine Entpolitisierung der GWA feststellen, wie Becker (2016) konstatiert. Er ist der Ansicht, dass auch ein zunehmend pädagogischer Auftrag an die Soziale Arbeit in und mit dem Gemeinwesen zu beobachten ist, z. B. durch das Handlungsprogramm „Gesundheitsförderung im Quartier“. Hier soll die Förderung von Bewegung und Ernährung unterstützt werden, wobei eine sozialraumorientierte Gesundheitsförderung deutlich darüber hinausgehen und die Veränderung ungünstiger Bedingungen, z. B. schlechte Wohnbedingungen, hohe Arbeitslosigkeitsraten oder starke Verkehrsbelastungen, mit in den Blick nehmen würde (vgl. Becker 2016: 15). Das Spannungsfeld zwischen Grassroots und Fremdbestimmung war und ist ein aktuelles Thema für Akteur*innen im Kontext der Gemeinwesenarbeit.

\section{Herausforderungen und Perspektiven}

Die aktuellen gesellschaftlichen Entwicklungen erfordern mehr Aufmerksamkeit für das soziale Miteinander in der Gesellschaft, erweiterte Handlungsoptionen im Umgang mit heterogenen Nachbarschaften und neue Möglichkeiten für Menschen, denen Teilhabe in verschiedenen Bereichen vorenthalten wird. Gemeinwesenarbeit bedeutet Demokratieentwicklung durch basisnahe Teilhabe-, Bildungsund Partizipationsmöglichkeiten für alle. Damit Gemeinwesenarbeit (in heterogenen Nachbarschaften) wirken und der Brückenbau professionell unterstützt werden kann, gilt es, zentral gelegene, attraktive Begegnungsorte zu schaffen, die für alle Menschen gut und barrierefrei erreichbar und nicht ideologisch belegt sind (religions- und parteipolitisch neutral) sowie verschiedene Milieus ansprechen. In Nachbarschaften braucht es nicht zunehmend spezialisierte Angebote, sondern nichtkommerzielle Orte zur Begegnung und kreative Möglichkeitsräume. Durch diesen niedrigschwelligen Ansatz mit Anlaufstelle im Stadtteil werden für alle Menschen, unabhängig von ihrer Herkunft, ihrem Alter, ihrem Aufenthaltstitel oder ihrer Beeinträchtigung, verschiedene Teilhabe- und Bildungsangebote geschaffen, sodass die Menschen in ihrer Nachbarschaft Zugehörigkeit und Beteiligung erfahren können.

Für die Arbeit an einem guten und demokratischen Miteinander in zunehmend heterogenen Nachbarschaften braucht es entsprechende Rahmenbedingun- 
gen, eine angemessene, langfristige Finanzierung und geeignetes, multiprofessionelles und vielfältiges Personal. Eine Zusammenarbeit verschiedener Professionen (z. B. aus Stadtplanung, Sozialer Arbeit, Stadtsoziologie, Geografie, Ökonomie), die ihr spezifisches Wissen einbringen, erscheint angesichts der komplexen Gemengelage in Stadtteilen wichtig. Angesichts der zentralen Bedeutung sozialer Belange, der Ansprache aller Milieus und Gruppen vor Ort, kommt der Sozialen Arbeit und insbesondere den Gemeinwesenarbeiter*innen eine moderierende, aktivierende und strukturierende Rolle zu. Es gilt, Ressort- und Professionsbefindlichkeiten sowie Vorbehalte zwischen Theorie und Praxis zu überwinden, den fachlichen Austausch deutlich zu verbessern und die Forschung zu intensivieren.

Im Hinblick auf das Ziel lebenswerter, inklusiver Nachbarschaften greift ein „Management" der Menschen und Themen, wie es vielerorts praktiziert wird, zu kurz. Die Arbeit an einer nachhaltigen Gesellschaftsentwicklung und einem inklusiven Miteinander ist ein komplexer gemeinsamer Such- und Lernprozess. Vertrauen muss langfristig aufgebaut werden, um wirklich alle Menschen vor Ort einbeziehen, beteiligen und sie ermutigen zu können. Hierfür werden professionelle Brückenbauer*innen benötigt, die sich mit Selbstorganisationsprozessen, Empowerment, Chancen und Tücken des sozialen Kapitals, Gesprächsführung, Konfliktlösung, zivilgesellschaftlichen Prozessen, Netzwerkarbeit, antidemokratischen Prozessen etc. auskennen - Brückenbauer*innen, die sowohl mit bürgerschaftlich Engagierten zusammenarbeiten, als auch Brücken in Politik und Verwaltung bauen können. Da viele der Ankommenden aus kulturellen Kontexten stammen, in denen das soziale Miteinander eine bedeutende Rolle spielt, können wir diesbezüglich auch von ihnen lernen. Für ein soziales Miteinander in Nachbarschaften, aber auch für wechselseitige Lernprozesse der Menschen vor Ort, müssen lokal Möglichkeitsräume geschaffen werden, die von Gemeinwesenarbeiter*innen professionell begleitet werden.

\section{Literatur}

Alcántara, S., Kuhn, R., Renn, O., Bach, N., Böhm, B., Dienel, H.-L., Ullrich, P., Schröder, C. \& Walk, H. (2014): DELIKAT - Fachdialoge Deliberative Demokratie: Analyse Partizipativer Verfahren für den Transformationsprozess. Umweltbundesamt, Dessau. Aschenbrenner-Wellmann, B. (2014): Diversity als Gestaltungsaufgabe für Non-Profit-Organisationen. Beitrag für den Jahresbericht 2012/13 der Evangelischen Hochschule Ludwigsburg. In: Aschenbrenner-Wellmann, B. \& Fliege, T. (Hrsg.): Von der interkulturellen Öffnung zum Diversity Mainstreaming. Berlin: Logos Verlag.

Aschenbrenner-Wellmann, B. \& Ehrismann, R. (2014) Die Soziale Arbeit mit Migranten und Flüchtlingen. Rahmenbedingungen und methodische Herausforderungen. In: Soziale Arbeit (DZI) 10-11, 366-373. 
Beck, S., Kriemann, M. \& Luchmann, C. (2017) VHW Studie: Stadtmacherinnen und Stadtmacher. Teil 1 Zwischenergebnisse. Hrsg: vhw-Bundesverband für Wohnen und Stadtentwicklung e.V. Abrufbar unter: https:/www.vhw.de/publikationen/vhw-werkstadt/. Letzter Zugriff: 30.6.2017.

Becker, M. (2014): Soziale Stadtentwicklung und Gemeinwesenarbeit in der Sozialen Arbeit. Stuttgart: Kohlhammer.

Becker, M. (2016): GWA- Personalbemessung. Konstanz: Hartung-Gorre Verlag.

Bringt, F., Klose, B. \& Trube, M. (2014): Gemeinwesenarbeit und Demokratie. Mobile Beratung und Gemeinwesenarbeit als sozialräumliche Praxis einer menschenrechtsorientierten Demokratieentwicklung. E-Paper Heinrich Böll Stiftung. Abrufbar unter: https://www.boell.de/sites/default/files/2016-02-gemeinwesenarbeit_und_demokratie. pdf?dimension1=ds_menschenfeindlichkeit. Letzter Zugriff: $30.10 . \overline{2} 018$.

Bundesarbeitsgemeinschaft Soziale Stadtentwicklung und Gemeinwesenarbeit e.V. (2004): Positionspapier der BAG zum Programm „Soziale Stadt“. Abrufbar unter: http:// www.bagsozialestadtentwicklung.de/downloads\#bag-veroeffentlichungen. Letzter Zugriff: 15.3.2018.

Drilling, M. \& Schnur, O. (2012): Nachhaltigkeit in der Quartiersentwicklung - einführende Anmerkungen. In: dies. (Hrsg.): Nachhaltige Quartiersentwicklung. Wiesbaden: VS Research: 11-41.

Fehren, O. (2006): Gemeinwesenarbeit als intermediäre Instanz: emanzipatorisch oder herrschaftsstabilisierend? In: Neue Praxis (46) 6: 575-595.

Fehren, O. (2016): Soziale Arbeit und Stadtentwicklung aus einer intermediären Perspektive. In: Drilling, M. \& Oehler, P. (Hrsg.) (2016): Soziale Arbeit und Stadtentwicklung. 2. Auflage. Wiesbaden: Springer VS.

Karstedt, S. (2004): Linking Capital. Institutionelle Dimensionen sozialen Kapitals. In: Kessl, F. \& Otto, H.-U. (Hrsg.): Soziale Arbeit und Soziales Kapital. Zur Kritik lokaler Gemeinschaftlichkeit. Wiesbaden: VS Verlag: 45-62.

Landhäußer, S. (2013): Gemeinwesenarbeit und die Aktivierung von sozialem Kapital. In: Stövesand, S., Stoik, C. \& Troxler, U. (Hrsg.): Handbuch Gemeinwesenarbeit. Opladen: $154-160$

Leiprecht, R. (Hrsg.) (2011): Diversitätsbewusste Soziale Arbeit. Schwalbach: Wochenschau Verlag.

Lüttringhaus, M. (2011): Zusammenfassender Überblick: Leitstandards der Gemeinwesenarbeit. In: Hinte, W., Lüttringhaus, M. \& Oelschlägel, D. (Hrsg.): Grundlagen und Standards der Gemeinwesenarbeit. Weinheim: Juventa: 277-281.

Notz, G. (2012): Theorien alternativen Wirtschaftens. Stuttgart: Schmetterling Verlag.

Mau, S. (2017): Die Fliehkräfte des Sozialen. Gesellschaftlicher Zusammenhalt heute. In: Forschung \& Lehre 4.

Oelschlägel, D. (2013): Geschichte der Gemeinwesenarbeit in der Bundesrepublik Deutschland. In: Stövesand, S., Stoik, C. \& Troxler, U. (Hrsg.) (2013): Handbuch Gemeinwesenarbeit. Opladen: Verlag Barbara Budrich: 181-202.

Oelschlägel, D. (2016): Soziale Arbeit und Stadtentwicklung aus einer parteilichen Perspektive. In: Drilling, M. \& Oehler, P. (Hrsg.) (2016): Soziale Arbeit und Stadtentwicklung. 2.Auflage. Wiesbaden: Springer VS: 45-56. 
Oelschlägel, D. (2017): Zur Geschichte der Gemeinwesenarbeit. In: Bundesverband für Wohnen und Stadtentwicklung e. V. vhw FWS 4: 171-175.

Penta, L. (2007): Visionen brauchen Fahrpläne. In: Penta, L. (Hrsg.) (2007): Community Organizing. Menschen verändern ihre Stadt. Hamburg: Edition Körber Stiftung.

Putnam, R. D. (1993): Making Democracy Work. Civic Traditions in Modern Italy. Princeton.

Putnam, R. D. (2000): Bowling Alone: The Collapse and Revival of American Community. New York: Simon\& Schuster.

Rausch, G. (2017): „Democracy must begin at home ...“ - Handlungsoptionen der GWA gegen den Populismus. In: Blätter der Wohlfahrtspflege 4: 132-134.

Riede, M. (2016): Gemeinwesenarbeit als Brückenbauerin. Zur Neuauflage eines bewährten Konzepts in heterogenen Nachbarschaften. In: Soziale Arbeit (DZI) 9: 325-332.

Riede, M., Rothschuh, M., Stracke-Baumann, C. \& Zychlinksi, J. (2017): Gemeinwesenarbeit und Geflüchtete - Inklusive GWA in neuen Nachbarschaften. In: Riede, M. \& Noack, M. (2017): Gemeinwesenarbeit und Migration. Verlag Stiftung Mitarbeit, Bonn.

Riede, M. (2017a): Gemeinwesenarbeit und das Städtebauförderprogramm Soziale Stadt. Ein Beitrag zur Bestandsaufnahme. In: vhw FWS 4 / Juli - September 2017. Abrufbar unter: https://www.vhw.de/fileadmin/user_upload/08_publikationen/verbandszeitschrift/FWS/ 2017/4_2017/FWS_4_17_Gemeinwesenarbeit_und_Soziale_Stadt_M._Riede.pdf. Letzter Zugriff: $12.12 .201 \overline{8}$.

Rothschuh, M. \& Spitzenberger, E. (2010): Auf dem Weg zu handlungsbezogenen Theorien der Gemeinwesenarbeit. In: Gahleitner, S. B. et al. (Hrsg.): Disziplin und Profession Sozialer Arbeit. Entwicklungen und Perspektiven. Opladen \& Farmington Hills: Verlag Barbara Budrich: 77-93.

Schnee, R.: (2010) Mit Blick auf die Stadtteile: Mikroökonomie als Chance!? Auf: stadtteilarbeit.de. Abrufbar unter: http://www.stadtteilarbeit.de/themen/buergerbeteiligung/ 95-literatur/theorie/lok-oeko-gwa/316-mikrooekonomie-als-chance.html. Letzter Zugriff: 30.7 .2017 .

Spatscheck, C. \& Thiessen, B. (Hrsg.) (2017): Inklusion und Soziale Arbeit. Teilhabe und Vielfalt als gesellschaftliche Gestaltungsfelder. Opladen, Toronto: Verlag Barbara Budrich.

Stoik, C. (2012) Gemeinwesenarbeit und Sozialraumorientierung - ein ,entweder-oder“ oder ein ,sowohl-als auch“"? In: sozialraum.de (4) 1/2012. Abrufbar unter: http://www.sozialraum.de/gemeinwesenarbeit-und-sozialraumorientierung.php. Letzter Zugriff: 30.5.2016.

Stövesand, S. \& Stoik, Christoph (2013): Gemeinwesenarbeit als Konzept Sozialer Arbeit - eine Einleitung. In: Stövesand, S., Stoik, C. \& Troxler, U. (Hrsg.): Handbuch Gemeinwesenarbeit. Opladen.

Stövesand, S. (2018): Gemeinwesenarbeit als sozialraumbezogenes Konzept und Handlungsfeld. In: Kessl, F., Reutlinger, C., Maurer, S. \& Frey, O. (Hrsg.): Handbuch Sozialraum. Grundlagen für den Bildungs- und Sozialbereich Springer, Wiesbaden, 3. Auflage. (im Erscheinen; Oktober 2018).

Voss, Kathrin (2010): Grassrootscampaigning und Chancen durch neue Medien. In: Aus Politik und Zeitgeschichte APUZ 19/2010. 
Wurtzbacher, J. (2014): Soziale Stadtentwicklung und Gemeinwesenarbeit. In: Benz, B., Rieger, G., Schönig, W. \& Többe-Schukalla, M. (Hrsg.): Politik Sozialer Arbeit. Band 2: Akteure, Handlungsfelder und Methoden. Weinheim: Beltz Juventa.

Open Access Dieses Kapitel wird unter der Creative Commons Namensnennung 4.0 International Lizenz (http://creativecommons.org/licenses/by/4.0/deed.de) veröffentlicht, welche die Nutzung, Vervielfältigung, Bearbeitung, Verbreitung und Wiedergabe in jeglichem Medium und Format erlaubt, sofern Sie den/die ursprünglichen Autor(en) und die Quelle ordnungsgemäß nennen, einen Link zur Creative Commons Lizenz beifügen und angeben, ob Änderungen vorgenommen wurden.

Die in diesem Kapitel enthaltenen Bilder und sonstiges Drittmaterial unterliegen ebenfalls der genannten Creative Commons Lizenz, sofern sich aus der Abbildungslegende nichts anderes ergibt. Sofern das betreffende Material nicht unter der genannten Creative Commons Lizenz steht und die betreffende Handlung nicht nach gesetzlichen Vorschriften erlaubt ist, ist für die oben aufgeführten Weiterverwendungen des Materials die Einwilligung des jeweiligen Rechteinhabers einzuholen.

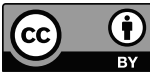

